\title{
La comunicación política en México: propuestas para su análisis
}

Luis Razgado Flores ${ }^{1}$

\section{Introducción}

\section{$E_{1}$}

avance del proceso democrático en México, sustentado, entre otros factores, por las reformas electorales y el respeto al voto, ha permitido que los procesos electorales cuenten con mayor independencia y confiabilidad tanto en su realización como en sus resultados.

A consecuencia de este hecho, los diferentes actores políticos que participan en las campañas electorales han empezado a considerar a la comunicación, el marketing y las técnicas publicitarias como importantes y efectivas herramientas que pueden ayudarles a presentarse ante los electores como opciones atractivas para ocupar cargos de elección popular.

Este fenómeno es reciente en la realidad política mexicana, ya que en los últimos 71 años el partido del Estado siempre había salido sistemáticamente victorioso en las elecciones presidenciales, aunque a finales del decenio de 1980 empezó a perder espacios de poder político.

En este nuevo escenario, que tiene su máxima expresión en la elección presidencial del año 2000, que representa la derrota del PRI, han surgido nuevos actores, prácticas e instancias que hace algunos años eran inexistentes en la realidad política mexicana, tales como: institutos de opinión pública, campañas basadas en marketing electoral, consultores de imagen, estrategias electorales sustentadas en la construcción de la imagen del candidato, debates entre candidatos y definición de estrategias discursivas de los candidatos.

1. Universidad Autónoma Metropolitana-Xochimilco, correo electrónico: razgado@terra.com.mx. 


\section{El vínculo entre medios, democracia y política en México}

El primer paso para iniciar un análisis fundamentado sobre la comunicación política en México, es elaborar un contexto del sistema de medios y el sistema político mexicano.

Hablar sobre democracia en nuestro país es referirse a un proceso de transición política que se ha venido gestando a partir de una serie de reformas electorales; de la creación de espacios autónomos que legitiman los procesos electorales y la conquista y apertura de espacios del poder público a la participación de diferentes corrientes políticas.

La conformación de los partidos políticos tiene una tradición ideológica que se gesta desde la Revolución mexicana y que con el tiempo ha permitido una serie de fusiones, transformaciones y escisiones. Estos cambios se han caracterizado por la existencia de vínculos históricos con grupos de interés político y económico en relaciones clientelares con organizaciones gremiales y, sobre todo, por la hegemonía política de un solo partido.

Anteriormente, partido y gobierno concentraban el manejo de la vida política y económica del país y tenían una importante influencia sobre otros ámbitos de la vida social y cultural. En el caso de los medios de comunicación, lo hacían a través de un control legal sobre ellos y de relaciones discrecionales entre los dueños de los medios y la clase política en el poder.

Si bien la prensa y la radio fueron, hasta mediados del decenio de 1960 , los medios de información fundamentales en la vida política nacional, la televisión se fue posicionando como el medio más importante de información y, a partir del decenio de 1980, se convirtió en el centro de la comunicación política y electoral en procesos de elección más competidos y con mayor participación. En esa época se gestaron una serie de acontecimientos políticos que dieron inicio a una etapa de democratización de los procesos electorales y la apertura para fuerzas políticas de oposición en espacios de participación política como el Congreso.

Por su parte, el sistema de medios de comunicación se ha transformado de manera significativa: la entrada en vigor del Tratado de Libre Comercio (TLC), abrió la posibilidad de inversión y participación de grupos extranjeros en la red de telecomunicaciones; el incremento en la publicación de periódicos tanto locales como nacionales, así como de estaciones de radio y televisión; la desaparición del monopolio televisivo comercial, ya que a partir de 1993 el monopolio de la televisión privada de señal abierta desapareció y con ello también el sistema de televisión federal; y los adelantos tecnológicos en materia de comunicaciones han influido para que se establezca una competencia entre medios a partir de sus contenidos. 
Sin embargo, es hasta las elecciones de 1994 que el uso y la importancia de la radio, y en especial de la televisión, se incrementa de manera notoria en las campañas electorales.

Paralelamente a estos cambios, es interesante observar que las instituciones políticas y las prácticas electorales que han surgido en los últimos quince años plantean un sistema pluripartidista que es dominado por tres partidos políticos mayoritarios (PRI, PAN y PRD) y distintos partidos llamados "pequeños", con lo que cambian las formas del quehacer político y las mismas negociaciones entre los actores.

En este sentido, cabe destacar que el apoyo a los partidos y sus candidatos descansa cada vez más en la opinión de los votantes y cada vez menos (en comparación al pasado reciente) en relaciones clientelares y corporativas. Por ello, es más evidente la importancia e independencia de un uso efectivo de la comunicación, un gran énfasis en la televisión como el medio para construir, mantener y acrecentar el apoyo y la personalización de la política a partir de los líderes de los partidos y personalidades destacadas.

Los aspectos interesantes de estas prácticas en México se han desarrollado con rapidez. Si bien han existido conflictos importantes entre los medios y los actores políticos, se ha iniciado un proceso de cambio entre la relación de los medios de comunicación, la política y la sociedad. Este cambio ha sido lento, ya que no ha seguido el mismo ritmo que los cambios políticos en el país, ni cubierto las necesidades comunicativas que estos cambios requieren, aunque, desde el surgimiento de prácticas más democráticas en los procesos electorales en México, se ha gestado un estrecho vínculo con los medios de comunicación, sus estrategias y prioridades.

En la actualidad, México tiene una sociedad más plural, participativa y demandante. Las instituciones políticas pasaron de una dinámica muy pasiva en su desempeño a un nuevo entramado de participación y reactivación de la competitividad y efervescencias partidarias. En este contexto, la influencia de otros actores sociales ha crecido, entre ellos los modernos medios de difusión masiva que se han convertido no sólo en escenarios, sino también en protagonistas de los cambios políticos y económicos.

Sin embargo, la relación entre medios y sociedad es todavía, en muchos sentidos, atrasada, desigual y autoritaria. Aunque es cierto que los medios están más abiertos a la expresión de los distintos actores que participan en los cambios de nuestrà realidad nacional, la representación que en ocasiones hacen de los acontecimientos, sobre todo la radio y la televisión, es superficial y descontextualizada.

Las similitudes y diferencias con otras democracias hacen interesante la exploración de la experiencia mexicana, en cuanto a que surge un sistema de comunicación política en medio de una era centralizada en los medios de comunicación, que incorpora gran parte de las estrategias comunicativas y de 
mercado. Además de lo anterior, llama la atención porque es un caso de estudio en el que se puede rastrear qué es lo que sucede cuando un sistema de comunicación política es construido en una sociedad y un sistema de gobierno que empiezan a tener una tradición democrática real en sus procesos electorales.

\section{¿Cómo surge la comunicación política contemporánea?}

La comunicación política es una de las manifestaciones dentro de la política y los medios masivos de comunicación que mayor inquietud han generado entre los estudiosos de la ciencia política, la psicología y las ciencias de la comunicación, entre otras especialidades, por ser considerada como una disciplina emergente.

En las últimas décadas, en especial en la de 1990, el enfoque en la comunicación política como objeto de estudio ha producido una diversidad de textos en los que se reflexiona sobre el papel de los medios en la política y sus efectos en la democracia.

El interés por estudiar esta disciplina se deriva del surgimiento de nuevos y complejos vínculos entre los actores políticos, los medios de comunicación modernos y la sociedad. Estos vínculos inciden, por una parte, en la relación tradicional entre actores y sistemas políticos al introducir nuevas variables en la reflexión del problema; y por otra, plantean cuestionamientos acerca del estatuto de lo público en las sociedades mediáticas.

Esto da por resultado una "nueva política" o una "nueva" forma de hacer política. En este sentido, la comunicación política se convierte en una realidad empírica, en un fenómeno visible, pero también en un concepto fundamental para entender los fenómenos políticos y las democracias contemporáneas.

Por ello, cabe preguntarse ¿a qué se debe la importancia de la comunicación en la política en los últimos 50 años? Las causas son diversas, pero a partir de un ejercicio de análisis y sistematización es posible considerar que estos cambios se originan a partir de cuatro factores:

1. La emergencia de la sociedad de masas.

2. El surgimiento de nuevos medios de comunicación en las democracias contemporáneas, en especial la televisión y los medios telemáticos.

3. La incorporación de prácticas y técnicas de la comunicación en la política.

4. Los cambios en las prácticas de los gobiernos democráticos.

Aunque sería posible considerar algunos otros factores, estos cuatro que mencionamos nos permiten, en primera instancia; entender la emergencia y centralidad de la comunicación que muchos autores identifican en las prácti- 
cas políticas actuales. ${ }^{2}$ Por ello es importante mencionar que en la realidad estos elementos están estrechamente relacionados, por lo que es difícil entender a uno sin el otro.

Uno de estos factores que me parece importante destacar es el que se refiere a la incorporación de prácticas y técnicas de la comunicación en la política, ya que el auge de los medios de comunicación masiva, entre los que destacan la televisión y las nuevas tecnologías de información, influye en la conformación de una nueva comunicación política, por lo que el rol que adquieren es fundamental dentro de esta dinámica. En ese sentido, David Swanson (1996: 125) advierte que:

A medida que las noticias televisivas se establecen como la primera fuente de información para el público, aumenta la rivalidad entre los medios de información y los funcionarios del gobierno por controlar el orden de los acontecimientos y por determinar cuáles serán considerados como asuntos de importancia y cómo serán presentados.

El planteamiento anterior advierte dos realidades a destacar: en primer lugar, una lucha por el establecimiento de una agenda de temas a tratar; en segundo lugar, la existencia de una relación de poder entre los políticos y los medios. Esta relación se ha dado con la prensa desde hace muchos años, pero lo que se plantea ahora es un vínculo no sólo de poder, sino de acercamiento y mezcla entre dos campos con sus propias dinámicas y lógicas: los grandes medios de comunicación y la actividad política.

Para dar cuenta del papel fundamental que la comunicación ha adquirido en la actividad política, basta observar empíricamente la propia evolución y crecimiento de los servicios de comunicación y asesoría de imagen. Hace unos cuantos años sólo existían oficinas de prensa, pero ahora se encuentran empresas que ofrecen servicios de imagen y comunicación, análisis de medios, diseño de estrategias y estudios de marketing, y en muchas ocasiones, sus servicios son considerados por algunos políticos como parte importante de la actividad proselitista, dejando en segundo plano actividades que por principio se consideran más propias del quehacer político.

La incorporación de prácticas y técnicas de la comunicación en la política genera cierta dependencia de los procesos de interés público con el mundo de la imagen (tales como: procesos electorales, demandas de grupos de interés,

2. Buena parte de los estudios recientes manifiestan esta inquietud, por mencionar algunos se citan distintos autores: Mancini, Paolo y David L. Swanson (1996); Monzón, Cándido (1996); Mouchon, Jean (1999); Muñoz Alonso y J. I. Rospir (1996, 1999); Alan Minc (1995); Wolton, Dominique (1998); Gilles Gauthier et. al. (1995), entre otros. En el caso mexicano, Raúl Trejo (1988) es un estudioso que ha publicado parte importante de la reflexión que se hace sobre el tema en México. 
actividades de los partidos políticos, relación entre actores políticos, debate y discusión de temas políticos, etcétera), por lo que se advierte un cambio aparente de roles: los periodistas pasan de ser testigos de la actividad política a actores de ésta, y por su parte, los líderes políticos aprenden técnicas de comunicación y persuasión e incorporan en sus equipos a especialistas de la imagen, el marketing y la publicidad (Monzón, 1996: 225).

Dentro de la nueva dimensión del rol de los medios en el desarrollo de la democracia, Cándido Monzón plantea dos ideas que dan cuenta de este cambio: a) la rendición de cuentas de los actos de gobierno debe darse con mayor rapidez y transparencia; y, b) los partidos políticos están perdiendo peso en la escena política, dando paso a la popularidad e imagen de sus líderes. Por lo tanto, concluye que "todos estamos envueltos en un nuevo espacio público dominado por la información" (1996: 226).

Si bien en todas las sociedades, como menciona Thompson (1998: 56), los seres humanos se dedican a la producción e intercambio de información y contenidos simbólicos, el desarrollo de los media ha transformado la naturaleza de la producción simbólica y el intercambio en el mundo moderno.

La emergencia de este aspecto de la comunicación mediática la convierte en una herramienta esencial para el político actual, ya que su uso supera la dependencia de la imagen para el desarrollo de una estrategia de campaña y de interacción entre gobernantes y gobernados. Como afirma Alan Minc, "para un hombre de Estado actuar y comunicar constituyen las dos caras de una misma realidad" (1995: 159).

Uno de los efectos de esta situación es abordado por David Swanson, quien plantea que nos encontramos ante ura "democracia centrada en los medios"3 que está revolucionando el mundo de la información y la política. En este sentido, el rol de los medios de comunicación ha cambiado de forma radical, al superar su concepción como simple canal đe eomunicación y convertirse en un actor potencial en el proceso político, en especial el electoral, con un peso específico y poder autónomo (Mancini y Swanson, 1996: 11).

Este fenómeno convierte a los medios en la primera fuente de información para el público dentro de una sociedad de masas y les otorga el poder de jerarquizar y controlar el orden y la forma en que presentan los acontecimientos. Esta capacidad de la prensa, la radio y, en mayor medida, de la televisión para confeccionar los mensajes y transmitirlos al público sugiere que los medios masivos están emergiendo como un centro de poder autónomo con importantes consecuencias para la política moderna. En este sentido, las

3. El término llamado también "mediocracia", al cual hacen referencia muchos autores, es planteado por David Swanson para referirse a la centratidad de los medios de comunicación en los procesos políticos, en especial en los periodos electorales, ya la relación entre los políticos y las instituciones políticas con sus electores. 
instituciones mediáticas y sus prácticas están indisolublemente vinculadas con las instituciones y prácticas de la democracia política. Un ejemplo de ello son las campañas para puestos de elección popular, que cada vez con mayor frecuencia se adaptan a las necesidades y los intereses de los mass media. ${ }^{4}$

Este tipo de operación ha "contaminado" la actividad política y a los políticos, develando una nueva forma de hacer política y de interactuar con los electores a gran escala; por lo que tanto los líderes como los candidatos y partidos políticos se plantean como necesidad el uso de los medios de comunicación de masas, de los expertos en comunicación y los sondeos y estrategias sofisticadas de comunicación para emplearlos y "convertirlos en diestros empaquetadores de sus ideas y personalidades para el consumo de los medios" (Swanson, 1996: 4).

\section{Comunicación política: una nueva perspectiva}

Otro antecedente es la incorporación de la mercadotecnia a la política. La mercadotecnia política tiene un peso consustancial en lo que los autores llaman "la democracia mediática", en donde muchos incluso identifican la nueva comunicación política con la mercadotecnia política (Gómez Fernández, 1999: 89).

En las tres últimas décadas, según Gómez Fernández (1995: 195-197), los grupos políticos, candidatos, partidos y gobierno preocupados por hacer llegar un mensaje comunicativamente más sofisticado y persuasivo (fundamentalmente en los momentos electorales y como técnica para conocer las posturas y preocupaciones de los votantes, a fin de formular estrategias encaminadas a conseguir el apoyo público), han desarrollado e incorporado prácticas que pertenecen básicamente a nuevas técnicas inspiradas en la promoción comercial. Esta lógica hace ver al electorado como mercado y al político como producto.

Autores como el belga Gabriel Thoveron (1998) y los franceses Roland Cayrol y Philippe J. Maarek (1997: 87) conciben al marketing político como el "método global de la comunicación política" y emplean de forma intercambiable los términos comunicación política y marketing político, "puesto que los dos están en la actualidad indisolublemente unidos o en vías de estarlo". Si bien esta afirmación reduccionista sobre la comunicación política es muy

4. Un ejemplo en el caso mexicano, es la participación de los precandidatos a la Presidencia de la República en programas cómicos, bajo los formatos de espectáculo televisivo, con el objeto de "tener un acercamiento con sus electores y romper con la imagen de seriedad de los políticos". Los formatos de los debates y la actuación de los políticos en ellos están determinados más por demandas de construcción de una imagen televisiva, que por los contenidos en sí mismos. 
cuestionable, ayuda a explicar el auge del marketing político en los sistemas democráticos a partir del decenio de 1960 y la forma en que algunos autores identifican la comunicación política moderna con esta disciplina de la mercadotecnia.

El marketing es un proceso por el cual las compañías seleccionan a sus clientes, analizan sus necesidades y, con base en esta información, posteriormente desarrollan innovaciones al producto, su publicidad, precio y estrategias de distribución. En la política, su aplicación se fundamenta en los mismos principios, pero el análisis de las necesidades se centra en los votantes y ciudadanos; el producto se convierte en una combinación multifacética entre el político en sí mismo, su imagen y la plataforma política de la que el político es partidario. La combinación de imagen e ideología, una vez constituida de manera integral, se promueve y difunde a la audiencia apropiada.

En el caso de la política existe una proposición o promesa con cierto valor de cambio que el político ofrece a los ciudadanos votantes como pago por su apoyo; el costo o precio que se paga puede venir en forma de votos, apoyo económico a la campaña, incorporación al trabajo voluntario, e incluso, a través de la manifestación de simpatías y respuestas positivas sobre el político ante los encuestadores.

Actualmente, la capacidad para influir en la opinión pública tiene un cierto "valor en el mercado político", está cada vez menos en las manos de los políticos y más en los tecnócratas y estrategas de la imagen. Hoy en día los candidatos no necesariamente deben tener la aprobación de las élites del partido para acceder a una candidatura, incluso a la de presidente.

Entre los factores que pueden llegar a establecer la diferencia para ganar los puestos de elección popular y tener una buena campaña, están el tener acceso a la contratación de encuestas de opinión, a la vez que contar con un estratega de medios y especialistas en imagen y con un equipo estable de consultores. Pero esto no significa que cualquier político pueda "comprar" la candidatura por elección, si tiene acceso a estos medios. Existen muchos casos que demuestran que la efectividad y el triunfo de un candidato no dependen únicamente de la cantidad de dinero que se haya invertido, ni de la creatividad de su campaña.

En la política, a diferencia del marketing, no únicamente se trata de anticipar las necesidades y deseos de un mercado para tener éxito; el proceso es más complejo, dinámico e impredecible, ya que mientras que en el mercado o ámbito comercial la información de los productos con la que cuenta el consumidor proviene básicamente de la publicidad a través de revistas, prensa, radio y televisión, en la política el caso es distinto. La actitud de los votantes está en constante cambio, ya que pueden ser influenciados por los comentarios que generan los medios sobre la acción de los políticos, así como por las discusiones que se desarrollan en sus círculos cercanos sobre política. 
Ningún candidato tiene la garantía de ganar con el acceso y uso de la mercadotecnia política, pero cualquier candidato con suficiente soporte económico y una correcta estrategia de mercadotecnia política tiene garantizada una audiencia que va a escuchar lo que tiene que decir y ello incrementa sus posibilidades de triunfo.

Otro elemento que posteriormente está ligado con la comunicación política y la mercadotecnia política, es la aparición de los sondeos de opinión en el decenio de 1930, ya que su desarrollo acompañará a la comunicación política de manera fundamental.

\section{Cómo entender a la comunicación política}

Abordar la comunicación política como un tema de estudio es algo nuevo y por tanto no existen conceptos claros; entenderla como un espacio, una disciplina o un recurso plantea ambigüedades que no son sencillas de resolver. En este sentido, Cándido Monzón, al hacer una revisión de las distintas conceptualizaciones que se han hecho sobre este término, plantea que el investigador se enfrenta ante un problema difícil de resolver debido a la existencia de ambigüedades, imprecisiones y controversias, por lo que pretender encontrar un consenso conceptual y analítico entre los estudiosos del tema es una tarea casi imposible, sobre todo si se parte de enfoques distintos.

El problema básicamente radica, como lo mencionan Monzón (1999: 219) y Swanson (1996: 6), en que por una parte no existen acuerdos entre los estudiosos de la comunicación política sobre los contenidos y fronteras de la disciplina; y por otra, los investigadores, en su intento de avanzar en el entendimiento académico del tema, ofrecen nuevas ideas, descubrimientos y teorías, por lo que existen ideas divergentes relativas a sus orígenes, metas y fines. Lo que es cierto y puede observarse en la bibliografía, es que cada vez se elaboran más conceptos y tratamientos sobre este evasivo y complicado objeto de estudio.

Esta complicación que casi todos los autores especializados hacen notar, se debe básicamente a dos factores: por un lado, estamos ante un fenómeno que para ser comprendido y estudiado necesita ser abordado desde una perspectiva interdisciplinaria; y por el otro, tiene un objeto de estudio en constante cambio. Es decir que las estructuras políticas, el sistema de medios y la relación entre los distintos actores, así como la misma tecnología, están sujetos a una dinámica de constante transformación.

Otro problema que dificulta el consenso entre los estudiosos sobre la conceptualización de la comunicación política y su metodología de abordaje, es que los fenómenos que se estudian están en cambio permanente, ya que las nuevas formas de relación y representación mediática entre los actores políti- 
cos se adaptan constantemente, por lo que los esfuerzos y trabajos que se realizan para entender el fenómeno quedan rezagados en poco tiempo.

Parte de este mismo problema radica también en que el estudio de la comunicación política adopta formas diferentes en cada país, al reflejar en su estudio cuestiones como:

- La situación política, cultural y la relación medios-Estado que tiene cada nación.

- El sistema político y de medios con que se cuenta.

- La experiencia particular con la "nueva comunicación política" o con su "democracia mediática".

- La preferencia hacia planteamientos particulares del estudio sistemático de la política y los medios.

- El desarrollo democrático que se experimenta.

Cualquier transformación en alguna de estas variables genera modificaciones en la realidad del fenómeno que se llega a analizar y describir. Esta situación es constante, sobre todo en democracias en proceso de transición, en las que los cambios en legislación electoral, sistema de medios y correlación de fuerzas son muy dinámicos.

Propuesta de cuatro enfoques para abordar el estudio de la comunicación política

Atendiendo esta preocupación y basándome en la definición que distintos autores hacen de la comunicación política, se pueden identificar cuatro enfoques que abordan el estudio de la comunicación política.

El primer enfoque podría denominarse de "influencia" y lo exponen autores como Robert Meadow, que en su trabajo manifiesta una atención especial hacia los efectos de las actividades comunicativas en el funcionamiento de los sistemas políticos. Un segundo enfoque de "contenido" se concentra en identificar los contenidos de los mensajes y los actores de la comunicación política. El tercer enfoque de "persuasión" es el que reconoce como parte fundamental de la comunicación política a un conjunto de técnicas y procedimientos para influir en los ciudadanos políticamente, dentro de las cuales puede incluirse el marketing político y, finalmente, un cuarto enfoque de "actores" que propone, a diferencia de los demás, que más que un proceso existe una interrelación de elementos que dan vida a la comunicación política, en la que existen, entre otros actores: los medios, las organizaciones políticas, los políticos, los periodistas, los sondeos de opinión y los ciudadanos.

Estos cuatro enfoques, como una forma de clasificar las distintas propuestas de estudio de la comunicación política, evidentemente presentan algunos 
problemas y limitaciones en su diferenciación, ya que en primer lugar todas las definiciones aluden a un mismo fenómeno; y en segundo lugar, en todas las concepciones hay actores, influencias, interacción y una serie de elementos comunes e interdependientes. Sin embargo, lo que se plantea para constituir las diferencias radica en la identificación de los factores que se privilegian sobre otros, y en ese sentido es que pueden articularse estos cuatro enfoques o maneras de entender la comunicación política.

A continuación me referiré particularmente al enfoque que he denominado de actores, ya que es sobre éste que se basa la propuesta de análisis de la comunicación política en México. Me parece que este enfoque reúne los elementos analíticos que permiten identificar las relaciones que surgen entre los distintos implicados en la comunicación política.

\section{Enfoque de actores}

Dentro del enfoque de "actores", Brian McNair y Dominique Wolton confluyen en sugerir otra manera de investigar la comunicación política, concentrándose en la interrelación de varios elementos que en ella participan.

McNair (1999: 5) identifica tres actores, que son: organizaciones políticas, medios y ciudadanos. Las organizaciones políticas son colectividades sociales que incluyen partidos políticos, organizaciones sociales, grupos de interés (cámaras, sindicatos, entre otros) y el gobierno. Los medios son usados como un término genérico que incluye todos los modos, niveles y maneras de la comunicación mediada, y los ciudadanos son considerados como individuos o grupos sociales que tienen propósitos comunes.

Por su parte, Dominique Wolton (1995: 110) establece que la comunicación política moderna tiene una especificidad, la cual no puede hacer que entendamos a la comunicación política como toda comunicación que tiene por objeto la política, en donde aparentemente predomina la forma sobre el fondo.

El proceso de intercambio de discursos políticos entre una cantidad cada vez mayor de actores políticos, implica que cada vez más la política moderna y el espacio público pasen por los medios y las encuestas de opinión. A partir de esto, Wolton (1995: 115) define a la comunicación política como: "el espacio en que se intercambian los discursos contradictorios de los tres actores que tienen legitimidad para expresarse públicamente sobre política y que son los políticos, los periodistas y la opinión pública a través de los sondeos".

Ambos autores plantean que abordar el estudio de la comunicación política desde una perspectiva relacional proporciona un mejor entendimiento del fenómeno, ya que entran en juego diversos elementos y es posible excluir fenómenos que rodean la política pero que no entran dentro del juego de actores. Además, la mayor parte de los estudios sobre comunicación 
política estudian la influencia de los medios o de los sondeos o de los políticos, en relaciones con dos a dos, pero pocas veces plantean el estudio en una relación de tres.

Tanto Wolton como McNair identifican elementos o actores de la comunicación política muy parecidos. Ellos consideran que la comunicación política es un concepto fundamental para el análisis del funcionamiento de la democracia, ya que permite la interacción entre información, política y comunicación. Estos tres elementos poseen un discurso y están encarnados en lo que se podrá llamar "actores de la comunicación política" y son inseparables de la democracia contemporánea, lo cual implica que ésta no se puede entender sin esos elementos.

\section{Elementos para el análisis de la comunicación política}

El enfoque de actores que se propone parte de la idea de que la comunicación política evoca todo aquello que se vincula con la producción e intercambio de los discursos políticos que exponen los distintos actores y que reflejan los medios (Wolton, 1995: 28-29). Éste es un punto de partida sobre el cual se tendrán que ir planteando una serie de elementos fundamentales que constituyen este tipo de comunicación.

Parte de la especificidad de la comunicación política surge del peso de la comunicación en la política, esto significa que el enfrentamiento político se realiza en un marco comunicacional y se constituye como un espacio de confrontación de carácter público, en el que se da el debate de puntos de vista contradictorios, de acuerdo con un conjunto de reglas y con la finalidad de que a partir de esta exposición polémica de ideas el electorado elija y se produzca un vencedor.

Esto nos deja ver que en las democracias pluralistas hay un desarrollo en el intercambio de discursos, el cual no implica que se comunique mejor, aunque proporciona un espacio en el que se pueden oponer, confrontar, reconocer como rivales y luchar políticamente sin cuestionar el funcionamiento de la democracia.

La comunicación política como espacio de interacción entre actores

La comunicación política se establece a través de la interacción de los discursos entre actores que ocupan un lugar dentro de la democracia debido a la legitimidad que tienen para su funcionamiento. 
Autores como Wolton (1996) y McNair (1999) plantean la existencia de tres actores que poseen un discurso y que representan legitimidades de la democracia (la política, la información y la comunicación): políticos, medios y sondeos de opinion, respectivamente, u organizaciones políticas, medios y ciudadanos. En esta perspectiva la comunicación política se entiende a partir de una cantidad limitada de actores, que básicamente son los que tienen legitimidad para expresarse e interactuar.

Aunque no todos los actores tienen la misma legitimidad, son parte constitutiva de la comunicación política y de la democracia, y cada uno de ellos tiene diferentes discursos, legitimidades e intereses; de ahí que la comunicación política se convierta en un espacio de interacción y confrontación de discursos contradictorios, indispensable para la política y la democracia contemporánea.

En la exposición de los discursos se produce una tensión, ya que cada uno pretende interpretar la realidad política del momento desacreditando o excluyendo la visión del otro. Estos antagonismos entre los discursos de los diferentes actores hacen que exista una permanente tirantez entre ellos, situación característica de la comunicación política actual. Estas diferencias se originan a partir de que tanto los actores como sus discursos parten de intereses y modos de actuar distintos, por lo que su legitimidad como actores dentro del espacio de la comunicación política, con la política y con la comunicación, es distinta.

Legitimidad y conformación de los actores que intervienen en la comunicación política

1. Actor político: el primer elemento de la comunicación política es el actor político, entendido como aquellos individuos que aspiran, a través de organizaciones e instancias institucionales, a influir en el proceso de toma de decisiones. Esta actividad la pueden realizar a través de organizaciones políticas que se constituyen a su vez como actores políticos, que son los partidos políticos, el gobierno y organizaciones no partidistas, entre las cuales existen grupos de presión y organizaciones gremiales, entre otros.

El discurso de los actores políticos está enfocado a la ideología y la acción, es decir, se trata de construir mensajes que justifiquen su actuación, sus decisiones, la manera que tienen de interpretar la realidad y la forma en que desean ser vistos por el público. En su discurso, su legitimidad resulta de la elección, y esta situación es lo que los hace ingresar en el espacio de la comunicación política, aunque no es el único camino. Para este actor, la función de la comunicación se traduce como una estrategia de persuasión: 
conseguir la adhesión de los demás actores a su causa o intereses, ya sean electores, políticos o periodistas.

2. Actor medios de comunicación: Este segundo elemento está constituido por las organizaciones de medios de comunicación y sus miembros, tanto periodistas como empresarios. La función de los medios en un sistema democrático es la de transmitir la comunicación política que se origina fuera del medio en sí, y también la de transmitir los mensajes políticos construidos por los periodistas. En este sentido, cabe mencionar que los medios no simplemente reportan de manera neutral e imparcial lo que ocurre en la arena política, existe una selección, jerarquización y tratamiento de la información, que convierte a los periodistas y a sus medios en mediadores y constructores de una realidad que es la que el público conoce y asume como verdadera.

Los actores políticos deben usar los medios para hacer llegar su mensaje al público objetivo. Los programas de políticas públicas, las campañas de los grupos de presión, los mensajes electorales e incluso los actos terroristas o de guerrilla tienen existencia política y un cierto potencial de comunicación efectiva, en la medida en que son reportados y recibidos por la audiencia como mensajes. Esto plantea una situación muy interesante que tiene que ver con las regulaciones y convenciones que permiten a los actores políticos tener acceso a los medios de comunicación.

Por su parte, la legitimidad de los periodistas resulta de su vínculo con la información, ya que tienen la capacidad, pero sobre todo cuentan con el "derecho" de acercarse y relatar los acontecimientos, produciendo así una visión de la realidad que se legitima socialmente. Si bien la información como valor es frágil en cuanto puede ser deformada, parte de su posición dentro de la comunicación política se debe a que los diferentes actores pueden ejercer su derecho y capacidad de crítica ante los acontecimientos y las acciones del poder. En este sentido, los medios de comunicación se interesan y "conmueven" en extremo ante el acontecimiento, siempre y cuando éste represente un conflicto.

3. Actor opinión pública-audiencia: Tanto Wolton (1996) como McNair (1999) plantean que existe un tercer actor dentro de la comunicación política, que es el que tiene como propósito toda comunicación y al cual se intenta persuadir: la audiencia.

Wolton plantea a la audiencia como un elemento más complejo en su conformación, que se representa a partir de los sondeos de opinión como la opinión pública; representación de la acción de la audiencia ante los mensajes de los otros dos actores.

McNair, por su parte, considera a la opinión pública, independientemente de su tamaño y naturaleza, como el elemento al que toda comunicación política pretende hacer llegar sus mensajes para impactar el comportamiento político de quien lo recibe. 
Ejes analíticos para caracterizar la comunicación política

De la reflexión hasta aquí expuesta, me parece importante resumir los elementos que se retoman del enfoque de actores sobre el cual se ha expuesto, por lo que es relevante rescatar dos elementos:

1. La identificación de los actores de la comunicación política como elementos constitutivos de la comunicación política y la interrelación que entre ellos se establece a partir de la interacción de su discurso. Actores que, como se dijo, no pueden estar únicamente representados o materializados por un solo tipo de actor, ya que eso representaría que cumplen una sola función, sino que, como lo señala el planteamiento de Mc Nair, en el caso de los políticos existen distintas categorías. Esta identificación resulta útil para emprender estudios de tipo empírico que produzcan información sobre las dinámicas entre los actores de la comunicación política en contextos particulares, ya que si bien existe una interacción entre éstos, su identificación permite separarlos y entender sus lógicas de acción.

2. De las ideas discutidas se proponen tres ejes analíticos, con los cuales se plantea caracterizar la comunicación política desde el enfoque de actores, que es el que me parece que ofrece una visión más amplia en el sentido del número de elementos que considera para entender la comunicación política contemporánea y define de manera más concreta el fenómeno de la comunicación política.

a) La interacción que se produce entre los actores políticos, los medios y los sondeos de opinión a partir del intercambio de sus propios discursos, ya sea para definirse o referirse a los otros actores.

b) El tipo de agenda que construye cada actor y las lógicas a las que obedece para privilegiar ciertos temas sobre otros.

c) El tipo de regulación o normas que se establecen de manera explícita e implícita para el enfrentamiento político.

A partir de las consideraciones hasta el momento expuestas se presenta una propuesta sobre cómo abordar el estudio de la comunicación política, retomando esta visión de actores y los ejes analíticos, la cual se aplica a un caso de estudio, y se presentan las conclusiones que dibujan un perfil de la dinámica de la comunicación política en México en épocas electorales. 


\section{Estrategia metodológica para el análisis de la comunicación política en México}

El objetivo es reconstruir esta lógica e identificar las estrategias y los recursos que los actores de la comunicación política — políticos (candidatos), medios de comunicación (prensa) y la opinión pública (sondeos de opinión)- utilizan dentro de un proceso de competencia política, en el que se generan condiciones de disputa donde se ponen en juego distintos tipos de recursos en el discurso, el desempeño mediático y la construcción de una imagen para llamar la atención de los electores.

\section{Unidades de estudio}

Las unidades de estudio son aquellas porciones de la realidad observada que tienen significación referente al fenómeno que interesa. Las unidades de estudio tienen características que les son propias, por lo que es necesario especificar distintos niveles que conforman los elementos de análisis. Determinar una selección de elementos para el análisis implica extraer elementos específicos de un universo de unidades posibles. A continuación se especifican las unidades de estudio que se consideraron en esta investigación.

1. Actor político: se identifica la identidad, función y características de los actores de la comunicación política en un caso específico. Son parte constitutiva del fenómeno que se estudia; de ellos se desprenden los discursos posibles y, por lo tanto, el universo probable del cual se compondrá una muestra de estudio.

2. Actor medios de comunicación: se especifica y caracteriza el medio o medios de comunicación que se incluyen dentro del estudio a partir del seguimiento que hacen de los actores políticos y sondeos de opinión.

3. Actor sondeos de opinión: se emplean los sondeos de opinión que aparecen en los medios seleccionados.

\section{Unidades de observación y análisis}

En los discursos de los distintos actores que intervienen en la comunicación política, se producen dinámicas en las que se confronta y construye el mensaje y la imagen que los candidatos quieren proyectar a sus públicos, en la que medios como la prensa y la televisión tienen un papel fundamental, al constituirse como un filtro entre el hecho y lo que se reporta de él.

A partir del discurso de cada actor se retoman las declaraciones de los candidatos y la forma en que los periodistas presentan la información, así 
como la inclusión de los sondeos, con el objetivo de iniciar un rastreo sobre las formas en que los candidatos diseñan su estrategia discursiva; cómo construyen al adversario; cómo se defienden ante las críticas de los otros y, finalmente, la información que la prensa privilegia en el espacio de la comunicación política.

Para el análisis se deben definir las siguientes unidades de observación de análisis que pertenecen a la unidad de estudio y son resultado de un trabajo descriptivo que permite entenderlas como un segmento específico de contenido que se caracteriza al situarlo en una categoría determinada.

\begin{tabular}{|c|l|}
\hline $\begin{array}{c}\text { Unidades de } \\
\text { observación y } \\
\text { análisis }\end{array}$ & $\begin{array}{l}\text { Las declaraciones hechas por los candidatos a los medios. } \\
\text { Las referencias que hacen los candidatos tanto a sus adversarios como a los } \\
\text { medios de comunicación, sobre el debate y los sondeos de opinión. } \\
\text { Los sondeos de opinión que aparecen publicados. } \\
\text { La forma en que los periodistas jerarquizan y dan prioridad a ciertos aspectos } \\
\text { de los actos de campaña de los candidatos y cómo se refieren a ellos. } \\
\text { La agenda temática que proponen los candidatos y los periódicos respecto al } \\
\text { debate y dentro de la campaña }\end{array}$ \\
\hline
\end{tabular}

Unidades de observación y análisis

- Las declaraciones hechas por los candidatos a los medios.

- Las referencias que hacen los candidatos tanto a sus adversarios como a los medios de comunicación, sobre el debate y los sondeos de opinión.

- Los sondeos de opinión que aparecen publicados.

- La forma en que los periodistas jerarquizan y dan prioridad a ciertos aspectos de los actos de campaña de los candidatos y cómo se refieren a ellos.

- La agenda temática que proponen los candidatos y los periódicos respecto al debate y dentro de la campaña.

Esta $s$ unid ades se sitú an en nive les de cate gorí as anal ític as gene rale $\mathrm{s}$ de estudio y a cada una le corresponde una serie de subcategorías de análisis específicas que particularizan el trabajo analítico y de clasificación de los discursos.

\section{Categorias analíticas}

Auto-representación del actor. Con esta temática de análisis se pretende observar cómo el actor se representa a sí mismo, qué propone como su estilo personal ante los medios y los electores y cómo trata de posicionarse ante el electorado, así como su manera de defenderse. Para esto se generan las siguientes subcategorías: 


\begin{tabular}{|l|l|l|l|l|}
\hline \multicolumn{1}{|c|}{ Actor } & \multicolumn{1}{|c|}{$\begin{array}{c}\text { Programa y } \\
\text { propuesta }\end{array}$} & $\begin{array}{c}\text { Ubicación } \\
\text { respecto al } \\
\text { adversario }\end{array}$ & $\begin{array}{l}\text { Presentación } \\
\text { de su persona }\end{array}$ & Partido \\
\hline $\begin{array}{l}\text { Se especifica el } \\
\text { candidato sobre } \\
\text { el cual se hace el } \\
\text { análisis. }\end{array}$ & $\begin{array}{l}\text { Se hace } \\
\text { referencia a los } \\
\text { planteamientos } \\
\text { que cada } \\
\text { candidato } \\
\text { expresa en sus } \\
\text { actos de } \\
\text { campaña y } \\
\text { discursos sobre } \\
\text { sus propuestas } \\
\text { de campaña e } \\
\text { ideologia. }\end{array}$ & $\begin{array}{l}\text { Qué posición } \\
\text { a los demás, } \\
\text { dentro del } \\
\text { contexto de la } \\
\text { campaña. }\end{array}$ & $\begin{array}{l}\text { Cuáles son los } \\
\text { atributos } \\
\text { personales que } \\
\text { cada candidato } \\
\text { dice tener en } \\
\text { cuanto a } \\
\text { experiencia, } \\
\text { honestidad, su } \\
\text { manera de ser, } \\
\text { etcétera. }\end{array}$ & $\begin{array}{l}\text { Cuáles son las } \\
\text { referencias que } \\
\text { hace cada } \\
\text { candidato sobre } \\
\text { su partido }\end{array}$ \\
\hline
\end{tabular}

Este nivel temático y las categorías que se proponen, permiten contar con información sobre la forma en que los candidatos se presentan ante el electorado y la manera en que construyen discursivamente su imagen.

Construcción del adversario. Un elemento importante dentro de la comunicación política es la manera en que los actores se relacionan con sus contrincantes políticos a través de su discurso. Es decir, los esfuerzos que hacen por representar al otro en un escenario electoral y construir y difundir la imagen de sus adversarios políticos, como parte fundamental para entender la interacción entre los actores políticos y los recursos de que se valen para hacerlo.

Para el desarrollo de este elemento temático existen dos tipos de análisis: uno, que corresponde a los candidatos y la forma en que califican a su adversario; y otro, que rastrea lo que otros actores políticos dicen sobre los candidatos.

Qué dice el candidato sobre su adversario en cuanto a:

\begin{tabular}{|c|c|c|c|c|}
\hline Candidato & Su persona & Posición política & Asociaciones & Calificativos \\
\hline $\begin{array}{l}\text { Se especifica el } \\
\text { candidato sobre } \\
\text { el cual se } \\
\text { expresa el } \\
\text { adversario. }\end{array}$ & $\begin{array}{l}\text { Referencias } \\
\text { sobre la } \\
\text { personalidad del } \\
\text { contrincante } \\
\text { político y su } \\
\text { desempeño. }\end{array}$ & $\begin{array}{l}\text { Referencias } \\
\text { sobre la posición } \\
\text { política que } \\
\text { manifiesta }\end{array}$ & $\begin{array}{l}\text { Elementos, } \\
\text { características o } \\
\text { conceptos con los } \\
\text { que se asocia }\end{array}$ & $\begin{array}{l}\text { Tipo de } \\
\text { calificativos } \\
\text { empleados para } \\
\text { desacreditar al } \\
\text { contrincante } \\
\text { político. }\end{array}$ \\
\hline
\end{tabular}

El objetivo de esta categoría es valorar la forma en que los candidatos representan y reconocen al otro dentro de su discurso. Es decir, la manera en que argumentan y se oponen a su adversario político dentro de un espacio 
comunicativo a través del diálogo, empleando la descalificación del otro como recurso.

Referencias que otros actores hacen sobre los candidatos

\begin{tabular}{|l|l|l|l|l|}
\hline \multicolumn{1}{|c|}{ Candidatos } & \multicolumn{1}{|c|}{ Actores } & Tipo de actor & \multicolumn{1}{c|}{$\begin{array}{c}\text { Sentido de } \\
\text { su discurso }\end{array}$} & \multicolumn{1}{c|}{ Discurso } \\
\hline $\begin{array}{l}\text { Se especifica el } \\
\text { candidato sobre } \\
\text { el cual se hace la } \\
\text { referencia. }\end{array}$ & $\begin{array}{l}\text { Se enuncia el } \\
\text { nombre del actor } \\
\text { que emite el } \\
\text { discurso. }\end{array}$ & $\begin{array}{l}\text { Se clasifica el } \\
\text { tipo de actor por } \\
\text { el giro o función } \\
\text { que tiene }\end{array}$ & $\begin{array}{l}\text { Se clasifica el } \\
\text { discurso en } \\
\text { cuanto a la } \\
\text { función que tiene } \\
\text { con relación al } \\
\text { candidato al que } \\
\text { hace referencia, } \\
\text { ya sea de crítica, } \\
\text { apoyou otros. }\end{array}$ & $\begin{array}{l}\text { Se cita la } \\
\text { declaración sobre } \\
\text { la que se hace el } \\
\text { análisis. }\end{array}$ \\
\hline
\end{tabular}

Este cuadro permite identificar la función que otros actores de la comunicación política tienen y el tipo de intervención que realizan en la confrontación política entre los candidatos.

Enfoque de cada actor sobre los otros actores de la comunicación política. Ésta es la categoría analítica más amplia de las propuestas presentadas, ya que, de manera cruzada, trata de identificar la posición de cada actor de la comunicación política frente a los otros actores, así como lo que cada uno, de manera particular, refiere sobre el otro. Esto permite rastrear, de manera específica, la relación que guardan: el político con los medios y los sondeos; los medios con los políticos y los sondeos y los sondeos con relación a los políticos y los medios. Lo cual ayuda a determinar el tipo de interacción existente entre los actores y el peso que cada participante tiene dentro de la comunicación política y permitirá, en su momento, evaluar la influencia y los desequilibrios que puedan existir entre ellos.

Para trabajar esta categoría se propone una división en otras seis categorías específicas, a fin de dar cuenta sobre cada relación planteada. A cada categoría le corresponde una serie de subcategorías que en algunos casos son las mismas y en otros cambian de acuerdo con la función del actor en cuestión.

Representación de los actores políticos sobre los medios. Qué dice el candidato...

\begin{tabular}{|l|l|l|l|}
\hline De qué medios habla & $\begin{array}{l}\text { Qué función dice que } \\
\text { cumplen }\end{array}$ & $\begin{array}{l}\text { Cómo califica a los } \\
\text { medios }\end{array}$ & $\begin{array}{l}\text { Cómo dice ser tratado } \\
\text { por los medios }\end{array}$ \\
\hline
\end{tabular}


Representación del papel de los medios en la contienda electoral \begin{tabular}{|l|l|}
\hline Cómo califican su labor & $\begin{array}{l}\text { Cómo se definen ante los candidatos } \\
\text { yel proceso electoral }\end{array}$ \\
\hline
\end{tabular}

Proyección política de los candidatos en los medios

\begin{tabular}{|l|l|l|l|}
\hline \multicolumn{1}{|c|}{$\begin{array}{c}\text { Qué destacan } \\
\text { las notas }\end{array}$} & Descripción del acto & $\begin{array}{l}\text { Referencias sobre } \\
\text { los candidatos }\end{array}$ & \multicolumn{1}{c|}{$\begin{array}{c}\text { Agenda que } \\
\text { privilegian }\end{array}$} \\
\hline $\begin{array}{l}\text { Se determinan los } \\
\text { aspectos que } \\
\text { privilegian las notas a } \\
\text { partir de sus } \\
\text { encabezados. }\end{array}$ & $\begin{array}{l}\text { Se identifica la manera } \\
\text { en que el medio } \\
\text { reporta la } \\
\text { concurrencia y } \\
\text { emotividad con que } \\
\text { describe la campaña, } \\
\text { asi como otros } \\
\text { aspectos. }\end{array}$ & $\begin{array}{l}\text { Se seña cómo se } \\
\text { refiere el medio con } \\
\text { respecto a los } \\
\text { candidatos. }\end{array}$ & $\begin{array}{l}\text { Se detectan los temas } \\
\text { que se privilegian en } \\
\text { las notas. }\end{array}$ \\
\hline
\end{tabular}

Utilización de los sondeos en los medios

\begin{tabular}{|l|l|l|l|}
\hline Cómo los califican & $\begin{array}{l}\text { Con qué frecuencia los } \\
\text { mencionan }\end{array}$ & $\begin{array}{l}\text { Qué importancia } \\
\text { manifiestan tener }\end{array}$ & $\begin{array}{l}\text { Importancia en la } \\
\text { campaña }\end{array}$ \\
\hline
\end{tabular}

Utilización de los sondeos por parte de los candidatos

\begin{tabular}{|l|l|l|l|}
\hline Candidato & $\begin{array}{l}\text { Qué importancia } \\
\text { manifiestan tener }\end{array}$ & $\begin{array}{l}\text { Relación entre } \\
\text { candidato y sondeos }\end{array}$ & Cómo los califica \\
\hline
\end{tabular}

De qué hablan las encuestas. En este nivel, lo que interesa es rastrear la importancia que han adquirido los sondeos de opinión y lo que reportan de la opinión pública dentro del proceso electoral seleccionado para analizar la presentación de su discurso e identificar los elementos que incluyen dentro de él.

\begin{tabular}{|l|l|l|l|l|}
\hline Candidato & Qué miden & $\begin{array}{l}\text { Qué interesa sobre los } \\
\text { candidatos }\end{array}$ & Temática & $\begin{array}{l}\text { Cómo se validan a si } \\
\text { mismos }\end{array}$ \\
\hline
\end{tabular}

Al emplear este tipo de categorías en cada actor, es posible ir rastreando la interacción entre los distintos actores: cómo se manifiesta y evoluciona conforme avanza la campaña.

Determinación del discurso de los actores de la comunicación política. La comunicación política se caracteriza por la interacción de discursos que tienen un significado determinado, están dirigidos a un destinatario y ejercen algún tipo de influencia sobre él. No todos los actores detentan de una misma 
manera la forma de producir su discurso, ni tampoco poseen la misma capacidad para estructurar su mensaje.

En todos los casos, el discurso ${ }^{5}$ alude a formas de expresión humana, a objetivaciones expresivas, por lo que se puede hablar de discursos del vestido o de los espacios. Por otra parte, el discurso alude a ciertas tendencias de elaboración de mensajes, a la preferencia de ciertos recursos por encima de otros y al tratamiento de ciertos temas en lugar de otros.

Partiendo de la idea anterior, el discurso de cada actor que participa de la comunicación política está regido por condiciones sociales de producción específicas en las que tiene lugar y que le son constitutivas. Es decir que, a partir de la posición o lugar que el enunciador y el destinatario ocupan en la trama institucional de una sociedad dada, en un momento particular se decide lo que puede y debe ser dicho. Por ello, entender el discurso como un texto permite encontrar las huellas de las condiciones que lo rigieron y que limitan o acotan su sentido.

El discurso de los actores de la comunicación política está determinado por condiciones coyunturales, que a su vez generan otras producciones discursivas de diferente índole. Por ejemplo, la declaración de un candidato genera a su vez un discurso en el periodista y en otro candidato.

Discurso de los políticos. En cuanto al análisis del discurso de los candidatos registrado en la prensa, interesa básicamente destacar:

- Las declaraciones y pronunciamientos de los candidatos sobre ciertas situaciones y temas que abordaron en los diferentes actos proselitistas de sus campañas teniendo como testigos a los medios.

- Discursos de los candidatos registrados en las notas informativas y crónicas de los diarios.

A través de este tipo de aproximación al discurso de los políticos es posible caracterizar y determinar su agenda temática, al contar con elementos que permiten rastrear la frecuencia y jerarquía que dan a ciertos temas que incluyen en sus discursos, así como conocer más información sobre el tipo de interacción que se manifiesta con los otros actores, al igual que su participación en la comunicación política y la interlocución que buscan.

Discurso de la prensa escrita. El periódico puede ser asumido desde diversas perspectivas: como una organización profesional concentrada en la producción y comunicación periódica de una secuencia de temarios sobre la actualidad política, social, cultural y económica de un estado, región o país. Asimismo, de manera más compleja, puede ser identificado como actor de la

5. Para los fines de esta presentación me parece conveniente significar el acto discursivo como una selección de términos y una combinación de los mismos, a la vez que una
selección de temas. 
comunicación política, al considerarlo como un actor social inmerso en el sistema político que es puesto en relaciones de conflicto con otros actores y especializado en la producción y la comunicación masiva de relatos y comentarios acerca de los conflictos existentes entre actores de ese y otros sistemas.

Los medios de comunicación son los que crean el acontecimiento a partir de publicitar un hecho preexistente o previsto que convierten en noticia (De Foncuberta, 1997: 103). En este sentido, Verón (1981: 67) señala que el acontecimiento en sí es una especie de variable desconocida que los medios de comunicación van a construir, en primer lugar, a partir del material y la información con que cuentan.

Lo anterior plantea, como se venía previendo, un problema sobre la definición de un mecanismo para abordar la información con la que se va a trabajar. Por un lado, el discurso unitario que da cuenta del acontecimiento que se reporta, el cual se plasma en las notas informativas entre otros géneros periodísticos; por otro lado, el discurso mismo del candidato y la toma de posición y comentario que hace el periodista sobre lo que reporta. El discurso periodístico basa su labor en la acción de otros actores, en actuaciones ajenas que realizan los actores políticos mediante el uso de lenguajes políticos.

El periódico presenta un discurso que en buena medida es un discurso sobre discursos: sobre discursos ajenos - de los actores, de las fuentes, de los sondeos- rara vez íntegramente transcritos, normalmente citados de manera fragmentaria o presentados de manera sintética, pero siempre incorporados al discurso propio, que los interpreta y evalúa. Será pues, tarea del análisis diferenciar entre lo que se refiere directamente al discurso de los candidatos y lo relativo a la jerarquización e inclusión que el periodista hace con relación a los hechos que reporta y de los cuales toma posición y comenta.

Discurso de la opinión pública. El discurso de la opinión pública que se considera es el que se muestra a través de los sondeos, los cuales se entenderán como el resultado de encuestas publicadas en los periódicos. En este sentido, se sistematizará y analizará la interpretación y uso que de éstas hacen tanto políticos como periodistas, el tipo de información que interesa, y se pregunta a los encuestados. Por otra parte, se analiza el papel de los sondeos de opinión a nivel cuantitativo, al medir la frecuencia con que los sondeos aparecen y son parte del debate político en el periodo analizado.

La estrategia metodológica que he expuesto es una propuesta que se puede aplicar como una forma posible, entre otras, de abordar y analizar el problema de la comunicación política, aunque no es definitiva y no está terminada, pero surge a partir de una serie de consideraciones teóricas y de análisis coyuntural que retoman planteamientos desde el enfoque de actores, desarrollado por Wolton y McNair.

La idea para realizar este trabajo fue contar con un modelo de análisis cualitativo que permitiera analizar y formular los rasgos distintivos que desde 
la interacción discursiva de los actores se puedan identificar como parte de lo que hemos definido como comunicación política.

En este sentido, esta propuesta tiene por objetivo caracterizar los rasgos de la comunicación política en México a partir de la reconstrucción de la lógica de interacción y transacción entre los medios, los actores políticos y los sondeos de opinión a través de sus discursos y la identificación de las características de cada actor, en el marco de la transición política, así como las tendencias de comportamiento global de los medios.

Por ello, analizar y determinar estos aspectos permite, desde un nivel micro que puede ser un análisis de caso, entender los cambios y ubicar los rasgos característicos de la comunicación política en México.

Finalmente, cabe mencionar que ésta es una propuesta que me parece totalmente criticable y perfectible, pero puede ser un punto de partida para reflexionar sobre la comunicación política en México desde una perspectiva más sólida que el puro análisis político o publicitario, el cual, si bien es importante y orientador, deja de lado muchas implicaciones en la interrelación de los actores, la lógica de actuación entre ellos mismos y con la sociedad.

\section{Algunas conclusiones sobre las características de las campañas electorales}

Las conclusiones que a continuación se presentan son resultado del estudio que se hizo de un caso concreto, la elección de jefe de Gobierno del Distrito Federal en 1997. Las deliberaciones teóricas, metodológicas y contextuales que se han expuesto tienen el objetivo de apoyar el análisis de la comunicación política en México como un fenómeno en el que pueden identificarse formas recurrentes de interrelación para ejercer este tipo de comunicación en periodos electorales.

El estudio que se presenta es un análisis inferencial, en el que se interpreta el discurso de los actores de la comunicación política en torno a ciertas categorías y subcategorías ya discutidas, con la intención de establecer un orden en su significado y su función.

A través de la identificación, categorización y descripción del discurso de los contendientes a la jefatura de Gobierno del DF se ha logrado establecer un análisis empírico de la relación entre actores políticos, sus temas de interés y formas de interacción, así como de las estrategias recurrentes para la construcción de su imagen y los mecanismos que emplean para enfrentarse en la arena mediática como adversarios para ocupar este puesto público.

Las siguientes reflexiones son resultado de la interpretación de algunas de las preguntas que a nivel personal generaron dichas pistas e indicadores 
sobre la comunicación política en nuestro país. Los planteamientos que se exponen surgen del análisis realizado para este trabajo y no siguen un orden específico ni pretenden, en ningún momento, ser totalizadores, sino propiciar una mayor reflexión teórica y analítica sobre el fenómeno de la comunicación política en México.

Esta investigación se planteó la pregunta: ¿Es posible determinar atributos peculiares de la comunicación política en México, de modo que claramente se distingan otros tipos de comunicación y contextos? La respuesta es afirmativa. Es posible identificar relaciones y elementos recurrentes en el desempeño de los actores que permiten pensar que la forma en que se genera la interacción en un ámbito electoral entre candidatos, medios y público sí posee un perfil determinado.

Este perfil se desprende, entre otros, de los siguientes factores:

- La apertura de los medios de comunicación a los procesos electorales en México ha sido clave en la conformación de la comunicación política, ya que además de contar con una regulación, hoy puede constatarse que hay mayor cobertura de las campañas de los partidos de oposición. Ésta es una condición fundamental de la democracia que los propios actores reconocen.

- La existencia de instituciones que regulan de manera autónoma las elecciones y algunas de las formas de interacción entre los adversarios, han influido en la conformación de la comunicación política actual.

- Las reformas electorales de los últimos años han hecho que se cuente con un nuevo marco legal y, por consiguiente, nuevas reglas del juego político que influyen de manera fundamental en la interacción ccmunicativa entre los actores políticos y posibilitan una competencia política más abierta y real.

- El origen de los cuadros políticos en México está marcado por el monopolio del poder por parte del PRI, que por más de 60 años formó a una parte importante de la clase política del país, y, otra parte significativa, aunque con menor presencia, surgió de la izquierda y la derecha. Por tanto, al existir desprendimientos importantes del PRI, sus ex militantes se ven obligados a enfrentar cuestionamientos sobre su pasado político, y además, los mismos priistas están forzados a responder o, cuando menos, dar la cara por los errores de su partido. 
- El sistema de medios de comunicación en México, así como la relación entre éstos y el Estado han sido también parte constitutiva de la comunicación política. La desaparición del monopolio televisivo y el establecimiento de una nueva relación entre el Estado y los medios de comunicación, que ha pasado de un estrecho control y colaboración a una relativa autonomía, han permitido que los partidos de oposición tengan un mayor acceso a los medios que en el pasado reciente. ${ }^{6}$

- La incorporación de estrategias asociadas con la mercadotecnia comercial, así como la consolidación de los sondeos de opinión en México en la práctica política, en especial en épocas electorales, han incluido nuevas variables que conforman la interacción e interpretación de la realidad por parte de los actores de la comunicación política.

Las ideas anteriores son parte de los factores que están influyendo de manera fundamental en la conformación de las características de la comunicación política en México.

\section{Los actores de la comunicación política}

La figura de los candidatos es la parte central del proceso electoral, ya que en su persona se centran las expectativas y atención del electorado. Esta situación permite reafirmar el mito de que las elecciones se ganan por las personas y no por los partidos políticos y la plataforma política que se propone. Aunque esta afirmación resulta un tanto engañosa, ya que en México se cuenta con un sistema político en donde los partidos son el único medio legalmente reconocido para acceder a puestos de elección popular, lo que hace que exista un vínculo importante entre lo que representa el candidato y lo que representa el partido que lo postula.

En México los partidos políticos tienen mucho que ver en la conformación de la imagen de su candidato y no son únicamente un medio para acceder al poder, sino que se constituyen como parte del perfil de éste. Es decir que los partidos políticos se constituyen como parte fundamental de la imagen de los candidatos.

Ante el cuestionamiento de quién tiene mayor importancia, si la agenda o las personas en la construcción de los candidatos como opción política diferenciada, es posible señalar que la respuesta no se encuentra en los temas y la manera de abordar la solución a los problemas de la ciudad, sino en la

6. En este sentido existen mediciones que arrojan información sobre los cambios que en cobertura informativa se han ido modificando en los últimos diez años. El trabajo de Raúl Trejo (1999) es un análisis cuantitativo de la cobertura informativa de los últimos periodos electorales en México, en los que se muestran estos cambios. 
construcción que tanto ellos mismos como los medios hacen de su perfil político y personal, y su desempeño en los actos de campaña. Esto significa que lo que vuelve distintos a los candidatos es que el acento no está puesto en la agenda, sino en otras características periféricas, como su personalidad, su estilo o su formación, entre otras.

La personalidad de los candidatos es una de estas particularidades, ya que ellos mismos tratan de posicionarse como poseedores de una serie de propiedades como la coherencia, la honradez, la tolerancia, el respeto, el carácter y la experiencia, y cada uno dice poseer tales atributos de manera diferenciada $y$, en consecuencia, basa gran parte de su discurso en legitimarse como tal.

El perfil político, así como las estrategias para ubicarse con respecto de su adversario en la contienda electoral, constituyen una forma más de establecer diferencias, en relación con una estrategia de comparaciones y distinciones entre lo que representa el contrincante y él, como poseedor de los atributos positivos. Existe un ejercicio constante de distintividad entre los actores políticos, en donde la preocupación se centra en qué hacer y decir para que no se confundan con los otros, y la estrategia parece ser que es apelar a valores.

Los actores políticos constantemente en su discurso hacen referencia a su persona como poseedores de valores que los hacen la mejor opción. Se caracterizan al plantearse binomios de personalidad como estrategia, en donde, al no estar solos en la arena política, su caracterización adquiere valor con base en lo que el otro no es o no posee. Entre los valores que se mencionan de manera recurrente son:

a) Honestidad vs. corrupción.

b) Estabilidad vs. caos.

c) Experiencia vs. inexperiencia.

d) Tolerancia vs. intolerancia.

e) Priista vs. no priista.

Lo interesante será observar en trabajos posteriores si en cada elección estos valores cambian o permanecen de acuerdo al contexto y los participantes de la elección. Lo que queda claro es que en la comunicación política en México la forma en que se construye el actor político e interactúa en su discurso con otros actores y con la ciudadanía, es a partir de valores.

En la disputa política existen valores que le proporcionan credibilidad y legitimidad al actor y su discurso. En la campaña política analizada, uno de los elementos que más llaman la atención es la identificación de un capital político que los candidatos dicen tener, garantizar o proporcionar. Es decir, valores sobre los que gira gran parte de su discurso y con los que pretenden hacer creíble su propuesta de gobierno y su imagen como gobernantes. En las campañas políticas se hace referencia constante a características que tienen 
cierto aprecio por la ciudadanía y éstas se convierten en parte medular del accionar político y de los discursos.

Al cuestionarnos sobre cómo se construye la legitimidad del discurso político, nos encontramos que los valores construyen legitimidad; la congruencia, la experiencia, la tolerancia y la honestidad construyen credibilidad al discurso, pero el problema surge cuando un actor ilegítimo habla de valores legítimos, ya que eso hace que se anule. Y ese es el problema que todos los candidatos se enfrentan al ser cuestionados. La coherencia, en este sentido, es un elemento que adquiere un valor importante que es así reconocido por todos los actores de la comunicación política, además de apreciable. Para los actores políticos es algo que se tiene que demostrar que se posee.

Así como existen valores que intentan generar credibilidad a la imagen que se construye el actor político o mediático de manera diversa, existe un valor que cubre toda la campaña y que todos los actores políticos dicen garantizar. En la elección de 1997 la honestidad es, sobre otros valores, aquel actor que generó mayores expectativas y, en ese mismo sentido, la credibilidad en torno a "la honestidad" tuvo más posibilidades de triunfo. Mientras más se le crea al candidato más oportunidades de triunfo tiene.

Cárdenas, por el trato que tuvo con los medios, las expectativas que generó y la forma en que supo aglutinar en torno a su candidatura de honestidad en su gobierno, determinó en gran medida la simpatía por su candidatura y su triunfo el 6 de julio. Lo interesante será observar, en relación al mismo factor, de valor común, en otras campañas, en qué cambia.

En este sentido, se puede decir, desde una perspectiva histórica, aunque sin mucho sustento metodológico, pero que ayuda a entender esta idea, que en la elección presidencial de 1994 "la estabilidad" fue el valor que se privilegió sobre otros, y que en la pasada elección presidencial del 2 de julio de 2000 , donde el PRI pierde después de 71 años en el poder la Presidencia de la República, "el cambio" se convirtió en el valor que prevaleció. Aquel candidato que tiene la capacidad de hacer más creíble en su discurso la capacidad de cumplir esa expectativa, obviamente, entre otros elementos aunados a ello, es el que ve de manera más amplia sus posibilidades de triunfo que las de sus adversarios políticos.

En la campaña se manifiestan modelos de acción política, en donde existe una lucha por identificar y definirse en algún modelo por parte de los actores políticos. El presentarse como técnico, estadista, político, persona simple, prudente, etcétera, es decir, como alguien que a partir de un modelo se le pueden asociar una serie de características, se convierte en una estrategia que se manifiesta de manera regular en los discursos de los actores políticos.

Los resultados de los sondeos de opinión, tanto los que son hechos públicos como los que se realizan de manera interna por los actores políticos, inciden en la comunicación política, ya que se convierten en insumos o dispositi- 
vos que hacen variar el discurso de los actores políticos, orientan el sentido e interpretación del discurso, así como la forma en que se jerarquizan los temas de campaña. El papel de los sondeos de opinión por parte de los actores políticos es, en muchos casos, visto como un referente importante para la acción política.

La opinión pública, a través de los sondeos es asumida como la representación de actores no visibles, los ciudadanos y los electores, y por esta misma característica adquiere, en los reportes que hacen los medios de los resultados de las encuestas, un peso muy significativo en la comunicación política en general y en el ánimo de los actores políticos como un factor que influye en su discurso, en su actitud y en las perspectivas que se van generando a lo largo del periodo electoral.

\section{Interacción entre los actores}

El intercambio de mensajes entre los actores políticos adquiere una direccionalidad cuando reconocen a un interlocutor como blanco de sus descalificaciones, que regularmente es el candidato que según las encuestas posee las preferencias del electorado. Independientemente de las coincidencias ideológicas o programáticas, dentro del periodo de campaña electoral los adversarios políticos generan discursos que tienen el objetivo, en su contenido, de descalificar y disminuir la aceptación que tiene cierto candidato por parte del electorado. Esta estrategia de reconocimiento y acción responde a una dinámica de conflicto en la que, en este caso, los actores políticos se "unen" en su discurso contra otro actor, incluso desatendiendo sus propias diferencias y cuestionamientos.

La acción del actor político sobre el que se centra el flujo comunicativo, regularmente tiene dos caminos: cuando de las palabras se pasa a los hechos, al amenazar y en ocasiones interponer demandas judiciales por difamación; 0 , por otra parte, la desestimación de las acusaciones, acompañadas de una actitud de despreocupación y de exclusión de su agenda esas temáticas.

La política se manifiesta como una práctica de ocultamiento y revelación en la interacción de los discursos, ya que se expresa una dualidad entre lo que los políticos presentan como su imagen, su desempeño y su coherencia política, por un lado, y lo que aparentemente ocultan, pero que otros actores revelan. Existe una dinámica que se resume en que el candidato adversario toma el papel de: "lo que no muestra él, se los digo yo". Esta estrategia es constante y tiene en los periodos electorales un punto muy significativo cuando regularmente un candidato o actor político denuncia el comportamiento del otro a través de algún tipo de declaración espectacular. Esta forma de interacción tiene el objetivo no necesariamente de probar lo dicho, sino de cuestionar la imagen que se tiene del candidato, deteniendo la simpatía que 
pudiera existir hacia él, y esperar dividendos en las preferencias electorales. Estrategia que es reiterativa pero no muchas veces con los resultados esperados.

Esta actitud genera a su vez una réplica por parte del "agraviado", que regularmente es la de no probar su "inocencia" de manera contundente, éste se expone como víctima de una campaña de desprestigio sin sustento. En resumen, se puede decir que existe una constante tensión entre "lo que yo digo que soy y lo que dice el otro", y sobre eso se basa en mucho la comunicación política.

La comunicación política tiene como característica fundamental que el enfrentamiento se realiza a través de un marco comunicacional, que en el caso de este estudio se basa en los discursos que generan los actores. El lenguaje es el vehículo que candidatos, medios y sondeos de opinión articulan para relacionarse e interactuar con los otros actores y a su vez influir en sus decisiones políticas. En este sentido, pueden observarse ciertas características de interacción que existen en la comunicación política en México.

El lenguaje político se constituye como un elemento regulador de la interacción entre los actores, y regularmente aparece como un recurso que tiene que ser claro, cuando menos en la elección de 1997, por lo que queda excluido el uso de figuras retóricas como la metáfora o la ironía. Sin embargo, la situación es diferente para los medios de comunicación, en especial para la prensa, ya que ésta sí puede hacer uso de las distintas figuras retóricas para calificar a los actores políticos y relatar sus actos de campaña sin que exista ningún tipo de problema, ya que además puede aducir que obedece a un estilo periodístico. La caricatura política es un ejemplo de lo anterior.

La relación que se establece entre el actor político y los sondeos de opinión adquiere características peculiares según los resultados de éstas; si son favorables al actor político, regularmente su actitud es de aceptación y aun se asumen como un argumento incuestionable sobre la aceptación de la candidatura y la buena marcha de la campaña, incluso llegando a asegurar la existencia de tendencias irreversibles al triunfo. Pero el problema existe cuando los resultados le son adversos; en este sentido se puede identificar una posición sumamente crítica respecto a ellas. Las críticas se basan en aspectos metodológicos y también en su desaprobación de aquella información que resultaba opuesta a los puntos de vista propios y que no demostraba que el partido al que se pertenecía estaba ganando. La desconfianza también se genera a partir de la preocupación manifiesta sobre quién patrocina las encuestas, variable que incide en su desaprobación.

La comunicación política es un proceso de comunicación en el que intervienen distintos actores bajo reglas de interacción, donde cada uno intenta persuadir al otro en busca de influir en un público que se manifiesta a través de la opinión pública. Los objetivos son de diversos tipos, ya sean electorales o de presión, para tomar decisiones de carácter político y de convencimiento 
de acciones que influyen en el ámbito político social y económico en la vida de un país.

La comunicación política se puede entender a partir de la interacción discursiva que existe entre los candidatos, los medios y los sondeos de opinión, como un proceso de gran dinamismo y abierto al flujo de mensajes. En él se enfrentan discursos políticos opuestos que reciben el apoyo de alguno de los otros actores de la comunicación política: políticos (otros actores políticos diferentes a los candidatos, como líderes de los partidos políticos, legisladores, dirigentes gremiales o militantes entre otros), periodistas (a través del tratamiento que se hace de la información de los candidatos o línea editorial del periódico que favorezca a alguno de ellos), o bien por la opinión pública, a través de los sondeos (que se manifiestan en apoyo a alguno de los contendientes a partir de las simpatías que genera su persona o propuesta como opción electoral).

La victoria de un argumento sobre otro, si bien es muy frágil es más difícil de identificar, si es que existe, ya que cada actor de la comunicación política defiende sus posiciones y no acepta dentro de su discurso la derrota o el cuestionamiento a sus argumentos y actitudes. Por lo que la victoria se puede determinar a partir del resultado de la elección, que es a fin de cuentas lo que permite evaluar la efectividad de los discursos y las estrategias que los actores desarrollaron a lo largo de todo el periodo electoral.

La relación y el peso específico que cada actor (políticos, medios y opinión pública) posee en el espacio de la comunicación política inicialmente se plantea como una situación equilibrada, en donde cada uno tiene sus propios recursos y objetivos para interactuar con los demás y enfrentarse políticamente en un espacio de comunicación regulado. Pero al irse desarrollando la campaña se empiezan a manifestar desequilibrios entre los actores, situación que genera enfrentamientos inusitados. Existe una relación de fuerzas entre los discursos de cada actor, en donde cada uno intenta imponer su representación de la situación no sólo política sino también de los acontecimientos a los otros dos, tratando de obtener un dominio de la agenda de la comunicación política. El equilibrio que existe entre cada una de las lógicas de los actores de la comunicación política es muy frágil lo que provoca que a cada instante una esas tres lógicas pueda imponerse a las otras.

La ruptura que se da entre un actor político y el actor mediático produce una alianza entre el medio y otro actor político, llámese candidato, que pone en desventaja al primero. Esta situación genera desequilibrios entre los actores de la comunicación política, donde el favorecido son los medios, ya que son los que tienen los recursos para estar constantemente mediando entre el acontecimiento y el público, produciendo una visión parcial de la realidad al tener control sobre la inclusión, exclusión y jerarquización de la información que conforma su agenda. 
También el desequilibrio puede favorecer a la opinión pública a través de los sondeos, en relación al actor político, al existir la idea, que es muy recurrente entre los actores políticos bajo la lógica del marketing, de que no existe otra representación de la opinión pública que no sea la de los sondeos. Lo que hace que éstos se conviertan en el referente inmediato para la toma de decisiones y de la acción política de los candidatos.

Pero este desequilibrio también es favorable a los sondeos cuando existe una ruptura entre medios y opinión pública, ya que si los medios se presentan como "representantes de la opinión pública" - siendo una especie de portavoz crítico e implícito de la opinión, preguntando en nombre de ella-compiten contra ésta, que si bien son distintas, una cualitativa y la otra cuantitativa, los periodistas llegan de manera implícita a opinar y beneficiar a alguno de los candidatos, lo cual puede ser "descubierto y sancionado" por los otros actores, incluyendo al público. Lo que provoca que los periodistas, para ser más creíbles en su discurso, dejen su papel de "portavoces cualitativos" y retomen y asuman el discurso de los sondeos como representantes de la opinión pública para hablar de los candidatos, los políticos y de otros aspectos del periodo electoral.

Las reflexiones presentadas en este largo planteamiento de ideas y resultados de investigación pretenden auxiliar en el entendimiento del comportamiento y la lógica de las contiendas electorales, pero desde un enfoque comunicacional.

La recopilación de aportaciones de distintos campos teóricos relacionados con la comunicación y la política y el análisis presentado, en sí misma no pretende derivar en explicaciones ni conclusiones finales o únicas, pero sí se espera ofrecer al lector la oportunidad de establecer parámetros e indicadores útiles, que posibiliten una mayor comprensión de las características de la comunicación política en México.

Sirva, pues, lo hasta aquí expuesto, como punto de partida para elaborar nuevas hipótesis de trabajo, y como elemento que permita entender de manera más amplia y consistente los rasgos que adquiere la comunicación política en México.

\section{Bibliografía}

Del Rey Morató, Javier (1996), Democracia y posmodernidad. Teoría general de la información y comunicación política, Madrid, Ed. Complutense.

Ferry, Jean Marc y Dominique Wolton, et al. (1992), El nuevo espacio público, traducción de María Renata Segura, Barcelona, Gedisa. 
Ferry, Jean-Marc et al. (1998), "Las transformaciones de la publicidad política", en El nuevo espacio público, España, Gedisa.

Krippendorff, Klaus (1990), Metodología de análisis de contenido. Teoría y práctica, España, Paidós Comunicación.

Maarek, Philippe (1997), Marketing político y comunicación, España, Paidós. McNair, Brian (1999), An introduction to Political Communication, EE.UU., Routledge.

Minc, Alain, La borrachera democrática. El nuevo poder de la opinión pública, traducción de José Manuel López Vidal, Madrid, Temas de Hoy.

Monzón, Cándido (1996), Opinión pública, comunicación y política: la formación del espacio público, Madrid, Tecnos.

Mouchon, Jean (1988), Politica y medios: los poderes bajo su influencia, España, Gedisa.

Muñoz-Alonso, Alejandro (1990), Opinión pública y comunicación política, España, Eudema.

Newman, Bruce (ed.) (1999), Handbook of political marketing, EE.UU., Sage. Newman, Bruce (1999), The mass marketing of pollitics, EE.UU., Sague.

Nimo, Dan y Keith Sanders (1981), Handbook of Political Communication, London, Sage Publications.

Swanson, David y Dan Nimmo (1990), "New Directions in Political Communications", en $A$ Resource Book, London, Sage Publications.

Swanson, David L. y Paolo Mancini (1996), Politics, Media and Modern Democracy. An International Study of Innovations in Electoral Campaingning and Their Consequences, Wesport, Praeger.

Thompson, John (1988), Los media y la modernidad: una teoría de los medios de comunicación, España, Paidós.

Verón, Eliseo (1981), Construir el acontecimiento, Argentina, Gedisa.

Wolton, Dominique (1995), "La comunicación política: construcción de un modelo", en Jean-Marc Ferry, Dominique Wolton et al. (1995), El nuevo espacio público, Gedisa.

Wolton, Dominique (1998), "Las contradicciones de la comunicación política", en Jean-Marc Ferry et al., Comunicación y política, Gedisa.

Wolton, Dominique (1998), Penser la Communication, Francia, Champs Flammarion. 